\title{
A highly sensitive Leishmania infantum chagasi isolation method from bone marrow and peripheral blood of adults and children
}

\author{
Rodrigo Buzinaro Suzuki ${ }^{1,2}$, Aline Diniz Cabral ${ }^{1}$, Luciamare Perinetti Alves Martins ${ }^{2}$, Márcia Aparecida \\ Sperança ${ }^{1}$ \\ ${ }^{1}$ Center for Natural and Human Sciences, Federal University of ABC, São Bernardo do Campo, Brazil \\ 2 Discipline of Parasitology, Marilia Medical School, Marilia, São Paulo, Brazil
}

Key words: Visceral leishmaniasis; culture isolation; diagnostic; Leishmania infantum chagasi; peripheral blood.

J Infect Dev Ctries 2016; 10(11):1275-1277. doi:10.3855/jidc.8022

(Received 18 December 2015 - Accepted 5 April 2016)

Copyright (C) 2016 Sperança et al. This is an open-access article distributed under the Creative Commons Attribution License, which permits unrestricted use, distribution, and reproduction in any medium, provided the original work is properly cited.

Dear Editor,

Visceral leishmaniasis (VL) is the most severe form of a neglected tropical diseases group transmitted by phlebotomine sandflies harboring promastigotes forms of donovani complex Leishmania species with distinct eco-epidemiological patterns. The anthroponotic $L$. donovani in East Africa and India; and the zoonotic $L$. infantum in Europe and North Africa and in America after its introduction during European colonization, being named L. infantum chagasi [1]. Promastigotes regurgitated by sandfly vector into the mammalian host dermis are phagocytosed by monocyte cells lineage, replicating as amastigotes [2]. Infected macrophages migrate through lymphatic and vascular systems infiltrating lymph nodes (LN) bone marrow (BM), spleen (S) and liver, causing a strict fatal systemic disease, if untreated.

According to World Health Organization last review on global VL incidence, $90 \%$ of 0.2 to 0.4 million cases per year, worldwide, occur in six countries: India, Bangladesh, Sudan, South Sudan, Ethiopia and Brazil (90\% of American human cases); with a mortality rate of 10-20\% [3]. In endemic and new affected areas, early and accurate VL diagnosis are shown to decrease mortality rates [4].

VL classical diagnosis correspond to microscopic examination of amastigotes in BM, LN and $\mathrm{S}$ cells smears, Giemsa or Leishman stained, obtained through aspiration; a painful, invasive and risky procedure, principally in children. Accuracy of this method depends on the tissue examined, the quality of reagents used and the technician identification ability [5].
Serologic and nucleic acid based VL diagnostic methods present several drawbacks like cross reaction with others American endemic trypanosomiasis including tegumentar leishmaniasis species [6]. Presence of macrophages in peripheral blood (PB) makes culture isolation suitable, as showed for children and adult patients infected with $L$. infantum from Mediterranean basin [7-9]. Most prominent $L$. infantum culture isolation techniques from $\mathrm{PB}$ presenting high sensitivity depends on sample washing with culture medium and concentration, or isolation of peripheral blood mononuclear cells (PBMC) [7,9], which are hard to be performed in laboratory routine, principally due to contamination risk during sample manipulation. Low efficiency of $L$. infantum isolation methods are probably related to immune complexes mediated parasite lysis [10,9]. Therefore, by using a BM and PB cells extensive saline washing approach, we obtained a $100 \%$ sensitive $L$. $i$. chagasi isolation method directly from adult and children patients samples.

\section{The Study}

From January through August of 2015, 29 adults (10 female and 19 male, 22-77 years) and five children (one female and 4 male, 2-9 years) consecutive patients with clinical signs of VL, resident in the west region of the Brazilian São Paulo State, were attended at the Hemocenter of Marilia Medical School and submitted to routine VL tests. BM aspiration and PB with and without heparin or EDTA were collected for parasitological, serological and hematological examination, respectively. Serological test was 
performed through the Kalazar Detect (Inbios International, Seattle, USA), a rapid strip imunochromatography on the base of rK39 antigen. Optical microscopic detection of amastigotes was carried out by two specialized groups of three technicians in hematology and parasitology, respectively, on two different BM Leishman stained smears. For each smear, after technicians consensus, parasitological test was expressed as absence, presence, rare, and suggestive forms of amastigotes.

Subsequent to routine procedures, the discarding BM and PB samples of all 34 patients included in the study were used for $L$. $i$. chagasi isolation by culture. The protocol was approved by Marilia Medical School Human Experimental Ethical Committee (CAAE 50128015.5.0000.5413). From each patient, 500 microliter of $\mathrm{BM}$ aspirate and one to two milliliters of $\mathrm{PB}$, were centrifuged for 10 minutes at $4^{0} \mathrm{C}, 2000 \mathrm{xg}$. Liquid phase was discarded, and blood cells where washed with chilled phosphate saline (PBS) (Thermo Fisher Scientific, Walthan, USA) until clearance of the supernatant. Afterwards, one volume of M199 medium (Thermo Fisher Scientific, Walthan, USA) enriched with $10 \%$ of bovine fetal serum (Thermo Fisher Scientific, Walthan, USA) and $2 \%$ of filter sterilized male urine, were added to the washed cells following incubation at $28^{\circ} \mathrm{C}$ for 20 days or until promastigotes emergence.

Six adult and four children patients were positive for VL diagnosis (table 1). BM and PB cultures and parasitological tests presented agreement of $100 \%$. From these samples one was negative by serologic assay, confirming its manufacturer informed sensitivity of $90 \%$. High sensitivity and specificity of parasitological assay was probably reached due to the number of specialized technicians examiners, not always available in VL diagnostic services. BM and PB parasite isolation was generally obtained at the same day, except for one patient with BM being positive at day 10 and PB at day 20, probably due to increased amount of parasites in the former. L. infantum specificity of all isolates was confirmed by Sanger's sequencing of the V7V8 variable segment of trypanosomatides $18 \mathrm{~S}$ rRNA encoding gene, as previously described [11] [GenBank: KT762389KT762398]. L. i. chagasi negative patients presented hematologic alterations related to VL clinic and laboratorial characteristics, whose discussion is out of this report proposal.

\section{Conclusions}

Considering the simplicity, low cost and high accuracy of BM and PB L.i.chagasi isolation method, associated to the possibility to perform biological studies, asymptomatic infection investigation [12], and drug resistance test, we suggest its routine application. Adaptation of this method to the microculture scale using microplates [7] can also be tested in order to facilitate the visualization of parasites during isolation period. Also, to investigate asymptomatic L. i. chagasi infection, $\mathrm{PB}$ can be used for culture isolation, since $\mathrm{BM}$ aspiration is only recommended for symptomatic patients. Potential anthroponotic transmission of $L$. $i$. chagasi, supported by parasite PB circulation in adults and children, and by evidence of its principal vector Lutzomyia longipalpis urbanization and feeding in human blood [13], should be considered in VL control programs in South America.

Table 1. Demographic data and diagnostic methods outcome of Leishmania infantum chagasi infected patients

\begin{tabular}{|c|c|c|c|c|c|c|c|c|c|}
\hline \multicolumn{3}{|c|}{ Patients } & \multicolumn{2}{|c|}{ Parasitological exam } & \multirow{2}{*}{$\begin{array}{c}\text { Serology } \\
\text { ELISA }\end{array}$} & \multicolumn{4}{|c|}{ Culture isolation } \\
\hline Number & Gender & Age & Smear hematology & Smear parasitology & & $\mathbf{B M}^{*}$ & Day ${ }^{\S}$ & PBף & Day \\
\hline $\mathrm{A} * 03$ & M & 34 & Presence & Presence & + & + & 10 & + & 10 \\
\hline A10 & M & 47 & Rare & Rare & + & + & 12 & + & NA \\
\hline A21 & M & 60 & Presence & Presence & + & + & 10 & + & 10 \\
\hline A24 & M & 68 & Rare & Rare & + & + & 10 & + & 20 \\
\hline A28 & M & 29 & Suggestive & Inconclusive & + & + & 7 & + & 7 \\
\hline $\mathrm{A} 34$ & M & 75 & Suggestive & Suggestive & - & + & 10 & + & NA \\
\hline $\mathrm{C}^{\dagger} 08$ & M & 5 & Presence & Presence & + & + & 6 & + & 6 \\
\hline $\mathrm{C} 11$ & M & 2 & Presence & NA\# & + & + & 5 & + & 5 \\
\hline $\mathrm{C} 23$ & M & 6 & Presence & Presence & + & + & 9 & + & 9 \\
\hline $\mathrm{C} 29$ & F & 2 & Presence & Presence & + & + & 6 & + & 6 \\
\hline
\end{tabular}

*Adult, $\uparrow$ Children, $†$ Bone marrow, §Day at promastigotes emergence, Peripheral blood, \#Sample not available. 


\section{Acknowledgements}

This work was financed by Fundação de Amparo a Pesquisa do Estado de São Paulo (FAPESP), research grant 2012/20221-9, and post-doctoral fellowship to ADC 2013/26096-4.

\section{References}

1. Kuhls K, Alam MZ, Cupolillo E, Ferreira GE, Mauricio IL, Oddone R, Feliciangeli MD, Wirth T, Miles MA, Schonian G (2011) Comparative microsatellite typing of new world Leishmania infantum reveals low heterogeneity among populations and its recent old world origin. PLoS Negl Trop Dis 5: e1155.

2. Lang T, Lecoeur H, Prina E (2009) Imaging Leishmania development in their host cells. Trends Parasitol 25: 464-473.

3. Alvar J, Velez ID, Bern C, Herrero M, Desjeux P, Cano J, Jannin J, den Boer M (2012) Leishmaniasis worldwide and global estimates of its incidence. PloS One 7: e35671.

4. Oliveira JM, Fernandes AC, Dorval ME, Alves TP, Fernandes TD, Oshiro ET, Oliveira AL (2010) Mortality due to visceral leishmaniasis: clinical and laboratory characteristics. Rev Soc Bras Med Trop 43: 188-193.

5. Chappuis F, Sundar S, Hailu A, Ghalib H, Rijal S, Peeling RW, Alvar J, Boelaert M (2007) Visceral leishmaniasis: what are the needs for diagnosis, treatment and control? Nature Rev Microbiol 5: 873-882.

6. Ready PD (2014) Epidemiology of visceral leishmaniasis. Clin Epidemiol 6: 147-154.

7. Allahverdiyev AM, Bagirova M, Uzun S, Alabaz D, Aksaray N, Kocabas E, Koksal F (2005) The value of a new microculture method for diagnosis of visceral leishmaniasis by using bone marrow and peripheral blood. Am J Trop Med Hyg 73: 276-280.

8. Hide M, Singh R, Kumar B, Banuls AL, Sundar S (2007) A microculture technique for isolating live Leishmania parasites from peripheral blood of visceral leishmaniasis patients. Acta Trop 102: 197-200.

9. Maurya R, Mehrotra S, Prajapati VK, Nylen S, Sacks D, Sundar S (2010) Evaluation of blood agar microtiter plates for culturing leishmania parasites to titrate parasite burden in spleen and peripheral blood of patients with visceral leishmaniasis. J Clin Microbiol 48: 1932-1934.

10. Riera C, Fisa R, Udina M, Gallego M, Portus M (2004) Detection of Leishmania infantum cryptic infection in asymptomatic blood donors living in an endemic area (Eivissa, Balearic Islands, Spain) by different diagnostic methods. Trans R Soc Trop Med Hyg 98: 102-110.

11. Marcili A, Speranca MA, da Costa AP, Madeira Mde F, Soares HS, Sanches Cde O, Acosta Ida C, Girotto A, Minervino AH, Horta MC, Shaw JJ, Gennari SM (2014) Phylogenetic relationships of Leishmania species based on trypanosomatid barcode (SSU rDNA) and gGAPDH genes: Taxonomic revision of Leishmania (L.) infantum chagasi in South America. Infect Genet Evol 25: 44-51.

12. le Fichoux Y, Quaranta JF, Aufeuvre JP, Lelievre A, Marty P, Suffia I, Rousseau D, Kubar J (1999) Occurrence of Leishmania infantum parasitemia in asymptomatic blood donors living in an area of endemicity in southern France. J Clin Microbiol 37: 1953-1957.

13. Afonso MM, Duarte R, Miranda JC, Caranha L, Rangel EF (2012) Studies on the Feeding Habits of Lutzomyia (Lutzomyia) longipalpis (Lutz \& Neiva, 1912) (Diptera: Psychodidae: Phlebotominae) Populations from Endemic Areas of American Visceral Leishmaniasis in Northeastern Brazil. J Trop Med 2012:858657.

\section{Corresponding author}

Márcia Aparecida Sperança, $\mathrm{PhD}$

Universidade Federal do ABC - Center for Natural and Human Sciences

Rua Arcturus, 03 - Bloco Delta, Sala 226, Laboratório 105 CEP 09606-070 São Bernardo do Campo - São Paulo - Brazil Phone: 55.11.2320 6253/6187

Email: marcia.speranca@ufabc.edu.br

Conflict of interests: No conflict of interests is declared. 